\title{
Influence of Co addition on the magnetocaloric effect of FeCoSiAIGaPCB amorphous alloys
}

\author{
V. Franco, J. M. Borrego, and A. Conde \\ Dpto. Física de la Materia Condensada, ICMSE-CSIC, Universidad de Sevilla, P.O. Box 1065, \\ 41080 Sevilla, Spain \\ S. Roth \\ Leibniz Institut für Metallische Werkstoffe, IFW Dresden, Postfach 270016, D 01171 Dresden, Germany
}

(Received 9 January 2006; accepted 22 February 2006; published online 28 March 2006)

\begin{abstract}
The FeCoSiAlGaPCB alloys can be prepared as bulk amorphous materials, with outstanding mechanical properties and increased electrical resistivity. These features can be beneficial for their application as a magnetic refrigerant. The influence of Co addition on the magnetic entropy change of the alloy has been studied. This compositional modification displaces the temperature of the peak entropy change closer to room temperature, but reduces the refrigerant capacity of the material. For the Co-free alloy, the peak entropy change is increased with respect to a Finemet alloy containing Mo, but its refrigerant capacity is not enhanced. (C) 2006 American Institute of Physics. [DOI: $10.1063 / 1.2188385]$
\end{abstract}

Magnetic refrigeration is a field of research that has gained increasing attention, as it is considered an alternative to the gas compression-expansion cycle. At temperatures close to room temperature, rare-earth-based materials are among the most relevant ones. ${ }^{1-4}$ To display a big magnetocaloric response, two requirements have to be fulfilled: the material needs to exhibit a big magnetic moment and, also, a strong temperature dependence of magnetization close to the working temperature. This second condition, related to a magnetic phase transition, can be achieved in two different ways. Either by a first-order phase transition, which produces an abrupt temperature change of the magnetic moment and, therefore, a remarkable peak in the magnetic entropy change $\left(\Delta S_{M}\right)$ at the transition temperature, or by a second order phase transition, which causes a more smeared peak in $\Delta S_{M}$. However, the remarkable hysteresis that appears in some materials, associated to first order phase transitions, may reduce the actual efficiency of the cooling process. ${ }^{5}$ It has been pointed out, nevertheless, that in order to compare the characteristics of different materials as candidates for magnetic refrigerants, their refrigerant capacity (RC) in a reversible cycle, connected to the entropy absorbed by the refrigerant at the cold end of the cycle and its temperature span, should be used. $^{6}$

The search for low-cost materials for high-temperature magnetic refrigeration is a field of current interest. ${ }^{7-12} \mathrm{Re}-$ cently it has been shown that some soft-magnetic amorphous alloys are good candidates for this application, ${ }^{11}$ with a refrigerant capacity that is comparable to that of low-hysteretic Gd-based materials. ${ }^{4}$ It has also been shown that the nanocrystallization of the alloy, although broadening the $\Delta S_{M}$ peak, does not improve the RC of the material. Besides the peak entropy change, RC and material cost, there are other factors that should be taken into account for a material to be efficiently applied, ${ }^{12}$ such as mechanical properties, corrosion resistance, electrical resistivity, etc.

Multicomponent Fe-based bulk amorphous alloys, together with their promising magnetic properties, ${ }^{13,14}$ present outstanding mechanical properties. ${ }^{13,15}$ Pieces for application devices, with the final required shape, could be prepared by mold casting. For the $\mathrm{Fe}-(\mathrm{Al}, \mathrm{Ga})-(\mathrm{P}, \mathrm{C}, \mathrm{B}, \mathrm{Si})$ alloy series, cylinders with up to $3 \mathrm{~mm}$ in diameter have been obtained. ${ }^{13}$ Moreover, the high electrical resistivity associated to the noncrystalline character of the alloys is a beneficial feature for their application as refrigerant materials. However, there are few studies concerning the magnetocaloric response of bulk amorphous alloys. ${ }^{16}$ It has been shown that the Co addition to this family of alloys produces a decrease of its Curie temperature, $T_{\mathrm{C}}^{a m}{ }^{14}$ Therefore, this compositional change could be a way for tuning the $\Delta S_{M}$ peak temperature. In this work the magnetic entropy change of the FeCoSiAlGaPCB series is characterized, analyzing the influence of Co addition on the refrigerant capacity of the material.

Amorphous ribbons, $\sim 25 \mu \mathrm{m}$ thick and $\sim 10 \mathrm{~mm}$ wide, with nominal composition $\left(\mathrm{Fe}_{x} \mathrm{Co}_{y} \mathrm{~B}_{z} \mathrm{C}_{u}\right) \mathrm{Si}_{3} \mathrm{Al}_{5} \mathrm{Ga}_{2} \mathrm{P}_{10}$ (Table $\mathrm{I})$ were prepared by single-roller melt spinning. The amorphous character of the as-quenched alloys was checked by $\mathrm{x}$-ray diffraction. Previously to the measurements, samples were stress relaxed by thermal annealing in an Ar atmosphere at $675 \mathrm{~K}$ for $30 \mathrm{~min}$. The field dependence of magnetization was measured in a Lakeshore 7407 Vibrating Sample Magnetometer using a maximum applied field $H=15 \mathrm{kOe}$ with field steps of $50 \mathrm{Oe}$, for constant temperatures in the range $300-720 \mathrm{~K}$ in increments of $10 \mathrm{~K}$. The magnetic entropy change due to the application of a magnetic field $H$ has been calculated from the numerical approximation to the equation

TABLE I. Composition of the studied $\left(\mathrm{Fe}_{x} \mathrm{Co}_{y} \mathrm{~B}_{z} \mathrm{C}_{u}\right) \mathrm{Si}_{3} \mathrm{Al}_{5} \mathrm{Ga}_{2} \mathrm{P}_{10}$ alloys.

\begin{tabular}{crrrrrr}
\hline \hline Alloy & A & B & C & D & E & F \\
\hline$x$ & 70 & 56 & 43 & 29 & 17 & 5 \\
$y$ & 0 & 14 & 26 & 40 & 52 & 63 \\
$z$ & 5 & 6 & 8 & 9 & 10 & 12 \\
$u$ & 5 & 4 & 3 & 2 & 1 & 0 \\
\hline \hline
\end{tabular}




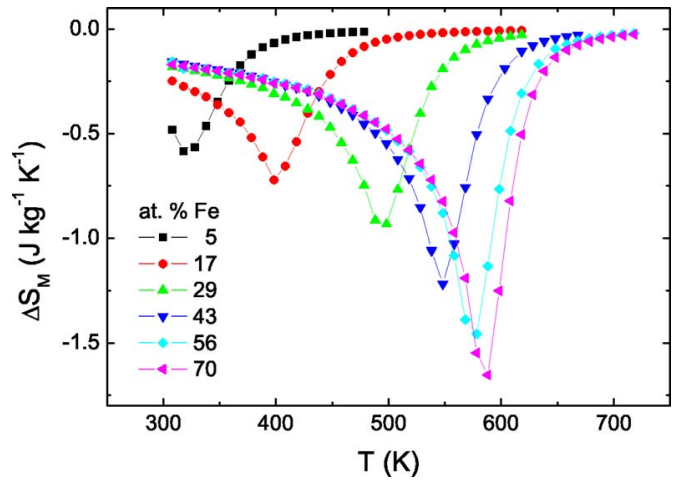

FIG. 1. (Color online) Temperature dependence of the magnetic entropy change for the different studied alloys. Lines are a guide for the eye.

$$
\Delta S_{M}=\int_{0}^{H}\left(\frac{\partial M}{\partial T}\right)_{H} d H
$$

where the partial derivative is replaced by finite differences and the integration is performed numerically.

Figure 1 shows the temperature dependence of the magnetic entropy change corresponding to an applied field $H$ $=15 \mathrm{kOe}$ for the different studied samples. There is a remarkable correlation between the $\Delta S_{M}$ peak temperatures $\left(T_{\mathrm{pk}}\right)$ of each sample and its corresponding $T_{\mathrm{C}}^{a m}$ calculated by the "kink point" method (Fig. 2). Although $T_{\mathrm{pk}}$ is shifted to temperatures closer to room temperature as the Co content in the alloy increases, the magnitude of the peak $\left(\left|\Delta S_{M}^{\mathrm{pk}}\right|\right)$ continuously decreases with increasing Co content (Fig. 2). This compositional dependence of $\left|\Delta S_{M}^{\mathrm{pk}}\right|$ is in agreement with the continuous decrease of the magnetic moment per transition metal atom as Co content increases in this series of alloys. ${ }^{14}$

The RC of the samples has been calculated by the Wood and Potter method ${ }^{6}\left(\Delta S_{M} \Delta T\right.$ in Fig. 3), either using the optimal cycle temperatures or imposing room temperature as the temperature of the cold reservoir (the cold and hot reservoir temperatures, $T_{c}$ and $T_{h}$, are plotted in Fig. 4 in both cases). To allow the comparison with published data for other materials, the product of the peak entropy change times the full width at half maximum of the peak $\left(\Delta S_{M}^{\mathrm{pk}} \Delta T^{\mathrm{FWHM}}\right.$ in Fig. 3), and the area under the $\Delta S_{M}(T)$ curves using the temperatures at half-maximum of the peak as the integration limits (Area in Fig. 3) have also been calculated. For the

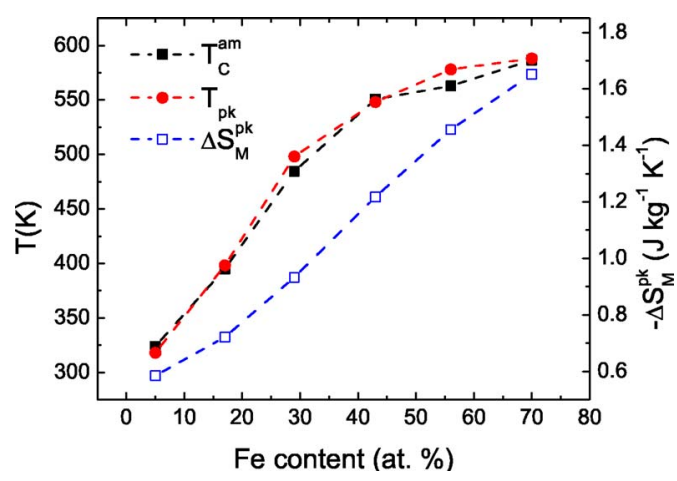

FIG. 2. (Color online) Compositional dependence of the Curie temperature of the alloys, the peak entropy change temperature, and the maximum magnetic entropy change. Lines are a guide for the eye.

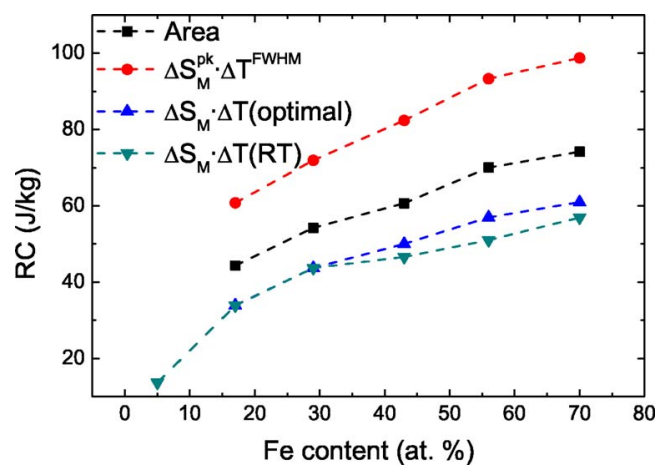

FIG. 3. (Color online) Compositional dependence of the refrigerant capacity (RC) calculated by three different methods (for the Wood and Potter method, RC has been calculated for the optimal reversible refrigeration cycle and for cycles departing from room temperature). Lines are a guide for the eye.

sample with the lowest $\mathrm{Fe}$ content, the RC for the optimal cycle could not be calculated, as the peak is too close to room temperature and $T_{c}$ (optimal) is below the available experimental range. As the Co content increases, the RC of the samples decreases. The minor differences in $\Delta S_{M} \Delta T$ for the optimal cycle and for that with $T_{c}$ at room temperature evidence that the best performance of these materials is achieved for a cold reservoir close to room temperature. Figure 4 shows the temperatures of the optimal cycles and those of the cycles with $T_{c}$ at room temperature. While $T_{h}$ displays no big differences between both cycles, $T_{c}$ (optimal) presents a more scattered character. This is related to the fact that the low-temperature part of the $\Delta S_{M}$ (Fig. 1) has a smaller temperature dependence and a minor change in the $\Delta S_{M}$ value corresponding to the selected temperatures of the cycles implies a relevant change in the associated temperature. As Co content increases, the temperature span of the cycles is reduced.

Although the Co addition in the FeCoSiAlGaPCB alloy series can be used to fine tune $T_{\mathrm{C}}^{a m}$ and, consequently, $T_{\mathrm{pk}}$, it decreases both $\left|\Delta S_{M}^{\mathrm{pk}}\right|$ and the RC of the material. The Cofree alloy presents a RC comparable to that of a Mo-Finemet alloy with a similar Fe content, ${ }^{11}$ although $\left|\Delta S_{M}^{\mathrm{pk}}\right|$ and the temperature span are bigger in the present case.

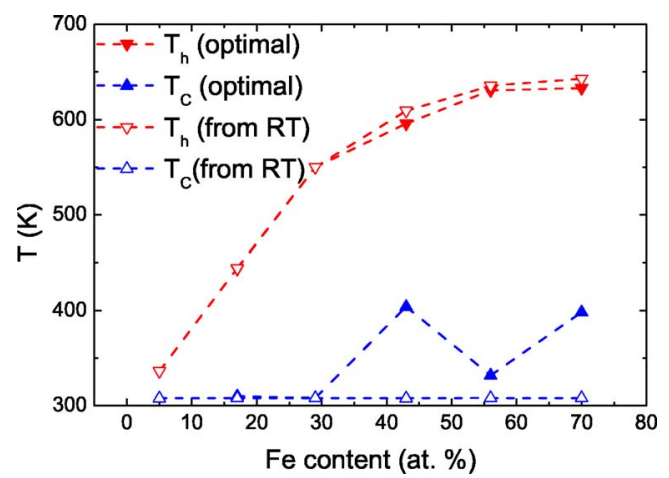

FIG. 4. (Color online) Compositional dependence of the temperatures of the cold and hot reservoirs for the optimal cycles (solid symbols) and for cycles departing from room temperature (open symbols). Lines are a guide for the eye. 
This work was supported by the Spanish Government and EU-FEDER (Project MAT 2004-04618) and the PAI of Junta de Andalucía.

${ }^{1}$ A. M. Tishin, in Handbook of Magnetic Materials Vol. 12, edited by K. H. J. Buschow (Elsevier, Amsterdam, 1999), pp. 395-524.

${ }^{2}$ K. A. Gschneidner, Jr. and V. K. Pecharsky, Annu. Rev. Mater. Sci. 30, 387 (2000)

${ }^{3}$ V. K. Pecharsky and K. A. Gschneidner, Jr., Phys. Rev. Lett. 78, 4494 (1997).

${ }^{4}$ V. Provenzano, A. J. Shapiro, and R. D. Shull, Nature 429, 853 (2004).

${ }^{5}$ E. M. Levin, V. K. Pecharsky, and K. A. Gschneidner, Jr., Phys. Rev. B 62, R14625 (2000).

${ }^{6}$ M. E. Wood and W. H. Potter, Cryogenics 25, 667 (1985).

${ }^{7}$ O. Tegus, E. Bruck, K. H. J. Buschow, and F. R. de Boer, Nature 415, 150
(2002).

${ }^{8}$ D. Wang, K. Peng, B. Gu, Z. Han, S. Tang, W. Qin, and Y. Du, J. Alloys Compd. 358, 312 (2003).

${ }^{9}$ I. Skorvanek and J. Kovac, Czech. J. Phys. 54, D189 (2004).

${ }^{10}$ S. Atalay, H. Gencer, and V. S. Kolat, J. Non-Cryst. Solids 351, 2373 (2005).

${ }^{11}$ V. Franco, J. S. Blázquez, C. F. Conde, and A. Conde, Appl. Phys. Lett. 88, 042505 (2006).

${ }^{12}$ E. Brück, J. Phys. D 38, R381 (2005).

${ }^{13}$ A. Inoue, Acta Mater. 48, 279 (2000).

${ }^{14}$ J. M. Borrego, A. Conde, S. Roth, and J. Eckert, J. Appl. Phys. 92, 2073 (2002).

${ }^{15}$ W. L. Jonson, Mater. Res. Bull. 24, 42 (1999).

${ }^{16}$ T. D. Shen, R. B. Schwarz, J. Y. Coulter, and J. D. Thompson, J. Appl. Phys. 91, 5240 (2002). 